\title{
The Challenges of Contact Tracing in a Case of Early Neonatal Sepsis with COVID-19
}

\author{
Mehmet Kenan Kanburoglu ${ }^{1}$ (D) $\cdot$ Ozgur Altuntas $^{1} \cdot$ Aysegul Copur Cicek $^{2}$ (D)
}

Received: 18 May 2020 / Accepted: 9 June 2020 / Published online: 22 June 2020

(C) Dr. K C Chaudhuri Foundation 2020

To the Editor: Contact tracing is one of the main components of slowing the chain of transmission during pandemics. This method entails screening the chain of contact in the infectious phase, reaching the people infected, monitoring them, and isolating those who are diagnosed for treatment. We present a neonate with COVID-19 and elaborate on the impeding factors that may arise while trying to ascertain the index case.

A 13-d-old female infant was admitted to the hospital with complaints of cough and fever. Upon physical examination, she had respiratory distress. Chest radiography showed bilateral interstitial infiltration and consolidation in the right upper lobe, and the CRP level was high. COVID-19 PCR result was positive.

We practiced permissive hypercapnia allowing a $\mathrm{pCO}_{2}$ level up to $60 \mathrm{mmHg}$ to prevent the spread of the virus and alleviate the complications of mechanical ventilation. She completed a 5-d course of ampicillin, piperacillin-tazobactam, azithromycin, and oseltamivir. She needed supplemental oxygen for three days and was discharged on day nine.

To find the source of transmission, we investigated all the people who may have had contact with the baby, including the family members and the hospital staff, by testing with nasopharyngeal COVID-19 PCR and for total IgG and IgM (Hotgen Biotech Co.). All results were negative. The sensitivity of the test was $77.1 \%$ and $74.3 \%$ for IgM and IgG, respectively [1]. The antibody response is closely related to disease severity; many asymptomatic people may have low antibody titers, which is inadequate for obtaining positivity on tests [2].

Mehmet Kenan Kanburoglu

kanburoglumk@outlook.com

1 Department of Pediatrics, Division of Neonatology, Recep Tayyip Erdogan University Medical School, Rize, Turkey

2 Department of Medical and Clinical Microbiology, Recep Tayyip Erdogan University Medical School, Rize, Turkey
Neonates have less developed immune system and hence may develop more severe disease [3, 4]. The infecting dose of the virus may be lower for neonates. This may explain why only the baby fell ill.

The use of computed tomography may have a role in identifying patients; nevertheless, it is ethically arguable. A total of $20 \%$ of patients may have no abnormalities on chest computed tomography [5].

In conclusion, we need to accomplish contact tracing thoroughly and with the most sensitive diagnostic tools available. Neonates may be more susceptible to acquiring disease if they encounter the same amount of viral load.

\section{Compliance with Ethical Standards}

Conflict of Interest None.

\section{References}

1. Rastawicki W, Rokosz-Chudziak N. Characteristics and assessment of the usefulness of serological tests in the diagnostic of infections caused by coronavirus SARS-CoV-2 on the basis of available manufacturer's data and literature review. Przegl Epidemiol. 2020;74: $113-32$.

2. Zhao J, Yuan Q, Wang H, et al. Antibody responses to SARS-CoV-2 in patients of novel coronavirus disease 2019. Clin Infect Dis. 2020. https://doi.org/10.1093/cid/ciaa344.

3. Lu X, Zhang L, Du H, et al. SARS-CoV-2 infection in children. N Engl J Med. 2020;382:1663-5.

4. Dong Y, Mo X, Hu Y, et al. Epidemiological characteristics of 2143 pediatric patients with 2019 coronavirus disease in China. Pediatrics. 2020;145:e20200702. https://doi.org/10.1542/peds.2020-0702.

5. Xia W, Shao J, Guo Y, Peng X, Li Z, Hu D. Clinical and CT features in pediatric patients with COVID-19 infection: different points from adults. Pediatr Pulmonol. 2020;55:1169-74.

Publisher's Note Springer Nature remains neutral with regard to jurisdictional claims in published maps and institutional affiliations. 